\title{
Review: self monitoring does not improve blood glucose control in type 2 diabetes mellitus
}

Coster S, Gulliford MC, Seed PT, et al. Self-monitoring in type 2 diabetes mellitus: a meta-analysis. Diabet Med 2000

Nov;17:755-61.

\section{QUESTION: What is the effectiveness of self monitoring for improving blood glucose control in patients with type 2 diabetes mellitus?}

\section{Data sources}

Studies were identified by searching Medline, EMBASE/ Excerpta Medica, the Index of Bibliography of Social Science, and the Cochrane Library with terms that included diabetes mellitus, self monitoring of blood glucose, and randomised controlled trials. Handsearches of diabetes journals, bibliographies of relevant papers, and the authors' personal reference files were scanned. Manufacturers and the British Diabetic Association were also contacted.

\section{Study selection}

English language studies were selected if they were randomised controlled trials (RCTs) that assessed the effectiveness of blood or urine glucose self monitoring for improving blood glucose control, as measured by glycated haemoglobin concentrations in patients with type 2 diabetes mellitus.

\section{Data extraction}

Data were extracted on study design, inclusion criteria, interventions, methodological quality, patients, and results.

\section{Main results}

8 RCTs (mean age range 54 to 65 y) that included 734 patients met the selection criteria. Study durations ranged between 3 and 12 months. The mean methodological quality score was 15 on a scale that ranged between 0 and 28 . Three studies were estimated to have sufficient power to detect differences in glycated haemoglobin concentrations between $0.5 \%$ and $1 \%$, and 4 studies were estimated to have sufficient power to detect differences $>1 \%$. 2 studies ( 1 that used fructosamine as the outcome measure and 1 that used cluster randomisation) were excluded from the meta-analysis. Metaanalysis of 4 studies (285 patients) showed that no difference existed between blood or urine glucose self monitoring and no regular monitoring for blood glucose control (weighted mean change in glycated haemoglobin concentration $-0.25 \%, 95 \% \mathrm{CI}-0.61 \%$ to $0.10 \%)$. Meta-analysis of 3 studies (278 patients) showed that blood glucose self monitoring and urine glucose self monitoring for blood glucose control did not differ (weighted mean change in glycated haemoglobin concentration $-0.03 \%$, CI $-0.52 \%$ to $0.47 \%$ ).

\section{Conclusion}

Blood or urine glucose self monitoring does not improve blood glucose control in patients with type 2 diabetes mellitus.

\section{COMMENTARY}

Clinicians who care for patients with chronic diseases are often faced with patients' lack of adherence to treatment recommendations. Many treatment regimens are complex and require adherence for long periods (eg, lifestyle modification). In general, isolated methods to improve adherence have been ineffective. ${ }^{1}$ Multidimensional strategies that promote and support patient self management are potentially more efficacious than one dimensional strategies. The assumption behind these strategies is that patient self management leads to improved and sustained patient outcomes.

Self monitoring allows patients with diabetes to make treatment decisions and evaluate their effect on glucose control with the support of a healthcare team. Patients who practice self monitoring become empowered to adjust their diet, exercise, and medications when they receive real time information from glucose monitors and test strips. In type 1 diabetes, glucose self monitoring is critical in guiding medication adjustments in multiple dose insulin programmes.

The review by Coster $e t$ al found that self monitoring in type 2 diabetes was ineffective in reducing glycated haemoglobin concentrations. Most of the studies included in this review tested self monitoring as the only or primary adherence improvement strategy. Thus, the best available evidence suggests that glucose self monitoring without a self management support programme is ineffective in reducing glycated haemoglobin concentrations.

Clinics that support a self management approach design the resources within their healthcare system and community to help patients to achieve their goals. Patients learn to use test values to evaluate their efforts. Patients who are unsuccessful with self management can find help from their family, social workers, educators, dietitians, nurses, and physicians. In this context, values obtained from test strips and monitors have meaning, and self monitoring may be effective. ${ }^{2}$

Victor Montori, MD

Mayo Clinic, Rochester, Minnesota, USA

1 Haynes RB, Montague P, Oliver T, et al. Interventions for helping patients to follow prescriptions for medications. Cochrane Database Syst Rev 2000;(2):CD000011.

2 McCulloch D, Price MJ, Hindmarsh M, et al. Improvement in diabetes care using an integrated population-based approach in a primary care setting. Dis Manage 2000;3:75-82.
Source of funding: NHS Research and Development Health Technology Assessment Programme.

For correspondence: Dr MC Gulliford, Department of Public Health Sciences, Guy's, King's and St Thomas' School of Medicine, King's College London, Capital House, 42 Weston Street, London SE1 3OD, UK. Fax $+44(0) 2078486605$. 